\title{
Analysis of visual field progression in glaucoma
}

Frederick W Fitzke, Roger A Hitchings, Darmalingun Poinoosawmy, Andrew I McNaught, David P Crabb

\begin{abstract}
Background-Despite the widespread use of computerised perimetry the diagnosis of visual field deterioration in following glaucoma patients over time remains particularly difficult. A new method of analysis using a novel graphical display of longitudinal field data is presented.

Methods-A linear regression model of the luminance sensitivity at each stimulus location against time of follow up transforms the quantitative data from a series of fields into a colour coded form which illustrates the spatial configuration of change to aid the interpretation of field loss. The method of analysis and the developed computer software (PROGRESSOR) is described. Comparison with STATPAC-2 glaucoma change probability analysis is given including levels of agreement between the techniques using series of fields of 10 eyes from patients with normal tension glaucoma.
\end{abstract}

Results-Examples of this new method compare well with STATPAC- 2 analysis. The level of agreement between the techniques to separate progressing from stable retinal locations is good (kappa $=0 \cdot 62 ; \mathrm{SE}=0 \cdot 04$ ). Conclusions-This new technique, which combines the change in perimetric sensitivity over time with colour coding of significant change into one image may provide an efficient method to detect true progression in glaucomatous field loss.

(Br f Ophthalmol 1996; 80: 40-48)

Inspecting a series of visual fields, generated by computerised perimetry for an individual eye, allows the determination of deterioration or stability. This assessment is a principal component of the analysis and monitoring of glaucomatous field loss. However, this diagnosis of progression is made difficult by the need to detect whether differences between subsequent fields are 'real' or merely 'noise' owing to patient response variability or other factors. The importance of this problematic task remains at the forefront of discussion on the definition and management of glaucoma. ${ }^{1-6}$

The need for methods to analyse extensive visual field data arises from the major development and more widespread use of computerised perimetry. Several methods have been proposed in recent years to aid the clinician in the diagnosis of true glaucomatous progression in a series of visual fields. Among those widely used are program STATPAC $-2^{7}$ for the Humphrey field analyser (Humphrey Instruments Inc, San Leandro, CA, USA) and the Delta program ${ }^{8}$ for the Octopus perimeter (Interzeag, AG
Schlieren-Zurich, Switzerland). They provide a battery of statistical functions to detect and evaluate change in perimetric sensitivity. These include probability of sensitivity change of individual field locations when compared with baseline and normal reference data. Other methods using data from all of the fields within a series have been developed and used. For example, regression and trend analyses have been applied to various estimates of sensitivity of the whole and parts of the field to assess progression. ${ }^{9-11}$ Other summary measures such as the visual field coefficient to compare progression between different treatment groups, ${ }^{12}$ sensitivity losses for the whole field and for quadrants, ${ }^{13}$ regression analysis of the mean defect value, ${ }^{14}$ and the rate of sensitivity loss and the number of locations which deteriorated have been used. ${ }^{15}$

The variability in threshold measurements and the methods based on summary estimates of the field as a whole or in part can frequently mask or falsely suggest progression especially when a small number of examinations are evaluated. For example, STATPAC-2 provides large numbers of locations in the probability change plots which indicate change for one field which are not sustained for the subsequent field. Moreover, constituent factors of field progression such as enlargement of existing scotomas, increased depth of defect, and appearance of new scotomas seem to vary for different subjects. ${ }^{116}$ Also, certain regions of the visual field may deteriorate at different rates. ${ }^{11} 1718$

In response to these outlined difficulties, analysis of the change in sensitivity at individual stimulus locations has recently been investigated. These pointwise methods include analysis of serial visual fields, using order of examination in conjunction with a mathematical model of the surface of the field, ${ }^{1920}$ and linear regression of the sensitivity at individual stimulus points against time of follow up. ${ }^{21-23}$ This latter technique has been shown to describe field loss masked by merely monitoring summary measures of field sensitivity. ${ }^{24}$ Moreover, a pointwise linear regression analysis of this type may provide a good prediction of future field status. ${ }^{25}$

The location of progression within the visual field has become of greater interest as this may provide indications of the spatial distribution of the pathology. Visualising the spatial locations of progression has been particularly difficult in previous methods for detecting progression of visual field loss as it requires inspection of a long series of fields to determine sensitivity loss combined with a separate series showing statistical significance and then appreciating their spatial relations within the visual field.

Science, Institute of
Ophthalmology, Bath
Street, London
F W Fitzke
D P Crabb
Glaucoma Unit,
Moorfields Eye
Hospital, London
R A Hitchings
D Poinoosawmy
A I McNaught
School of
Mathematics,
Actuarial Science and
Statistics, City
University, London
D P Crabb
Correspondence to:
F W Fitzke, PhD,
Department of Visual
Science, Institute of
Ophthalmology, Bath Street,
London EC1 9EL.
Accepted for publication
14 August 1995




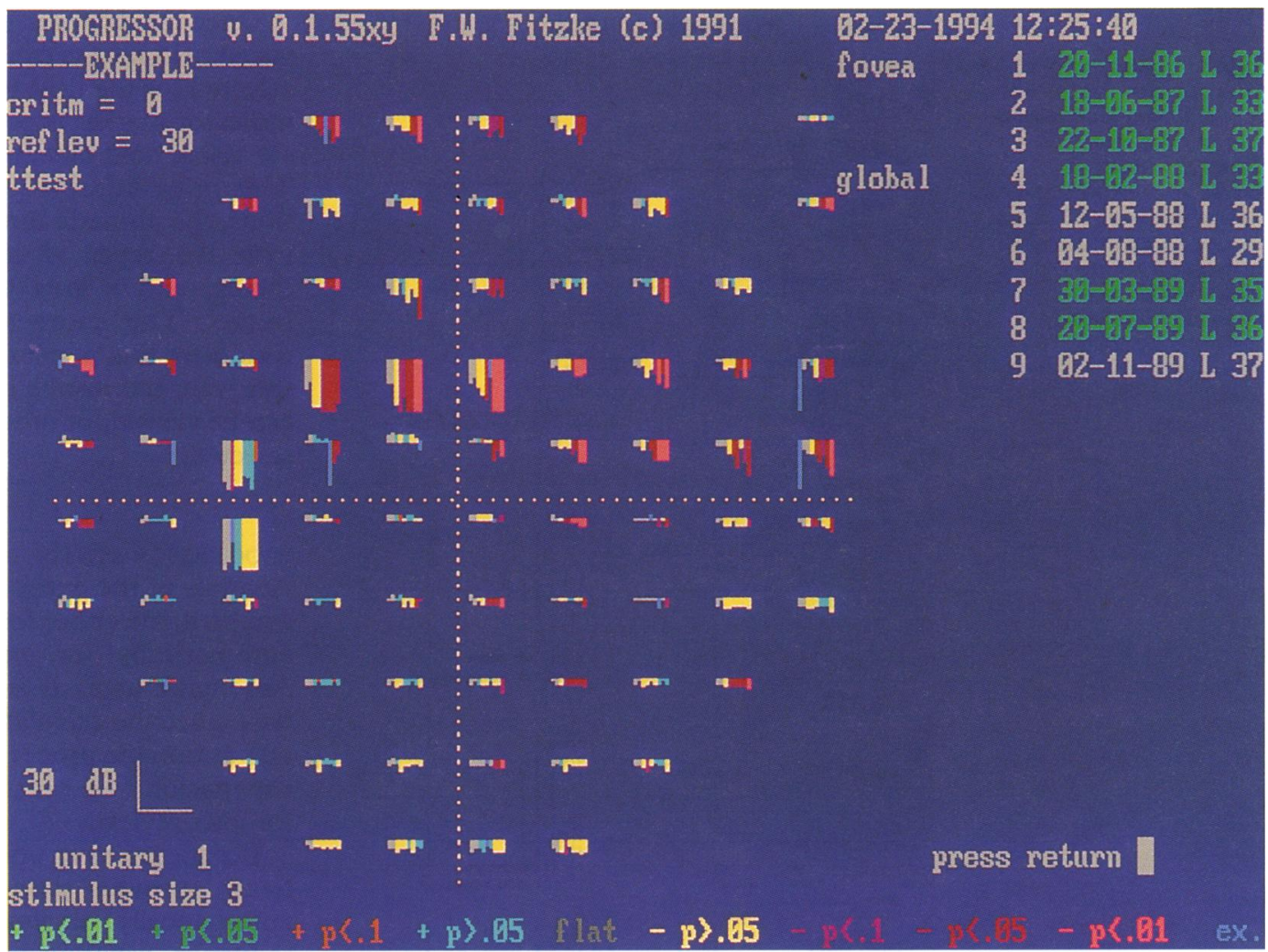

We have developed a new method for analysing serial visual field data implemented by a software package called PROGRESSOR. This takes advantage of a pointwise linear regression analysis $^{21}$ of luminance sensitivity values (decibels, dB) against time of follow up and uses all the data from all the fields within a series of examinations. It combines this analysis with a new form of graphical display which allows detailed inspection of the spatial and temporal characteristics of field loss in one colour coded figure. The software is used on a standard personal computer and provides database facilities with checks of data validity and visual field management. The programs have been tested extensively and used on hospital databases which consist of many thousands of patient visual fields. They have proved helpful in automating large parts of the analysis and in presenting the results in a form which is visually and intuitively easy to understand and interpret and to help detect significant progression of visual field loss.

In this paper we describe the PROGRESSOR software package including the data management facility. We also demonstrate the methodology that underpins the analysis of change in perimetric sensitivity and illustrate with patient examples. Moreover, the level of agreement between the pointwise linear regression method implemented by PROGRESSOR and the glaucoma change probability analysis from STATPAC- 2 is assessed. The agreement between the techniques to separate progressing from stable retinal locations is examined using series of fields from a sample of normal tension glaucoma patients.

\section{Materials and methods}

The programs are divided into several parts:
(1) preparation and maintenance of the patient visual field database

(2) checks of the database for internal consistency and errors

(3) displays of results of each visual field with numerical values, grey scales, and summary graphs of reliability indices

(4) statistical analysis of sequences of visual field results with graphical presentation and colour coding for significant progression

(5) summary reports, histogram plots, and comparison of results of patient groups.

The initial stage is the formation of the visual field database on the PC. This is done by transferring the Humphrey field analyser (HFA) visual field results to the PC hard disk directly from the HFA to the PC via a serial communications line or by reading the HFA floppy disk directly. Each patient's series of visual fields is grouped for easy access and patient groups can be further organised into divisions such as ocular hypertensive, normal tension glaucoma, or other selected groupings. When reviewing the results a patient group is selected and PROGRESSOR then collects together all the patient names which are in this group for display. The individual patient results are then available for viewing. The patient name is displayed along with full information about the visual fields.

The first time the database is formed, the program organises the visual field data into a unique patient group for each patient, to be held on the database. This preparation of the database is done largely automatically except when adding a new patient to the database for which a new unique patient identifier is created. Subsequently, the database management program provides extensive additional checks before analysis. Each unique patient identifier is checked for internal consistency 
Fig $1 B$

Figure 1 Analysis of a series of visual field results from a patient with normal tension glaucoma. $(A)$ Shows the results as displayed by PROGRESSOR. For comparison (B) shows the same data as described by output from STATPAC-2 'glaucoma change probability analysis'.

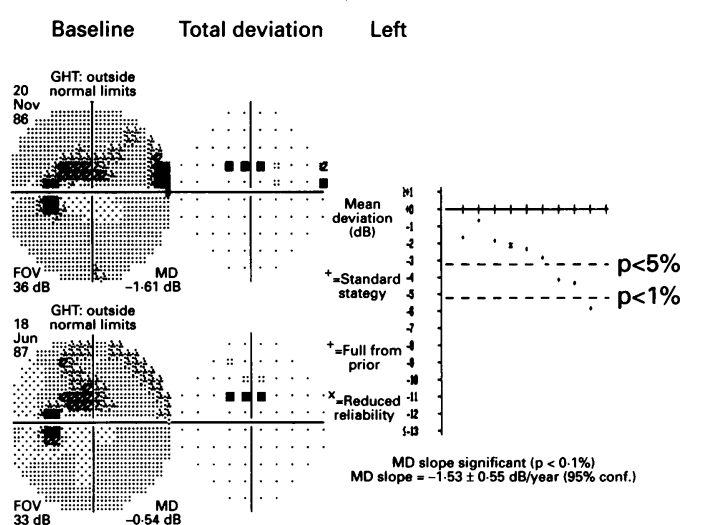

Once the database has been checked, patient visual field results can be reviewed. The date is selected for the field to be examined. When an indicator marker is positioned at the appropriate visual field date, the results can be viewed. This gives information about the test conditions, the numeric threshold values in $\mathrm{dB}$ units with the repeated measurements in parentheses, and a grey scale of the visual field results. The entire sequence of visual field results for that patient can easily be reviewed in this way, the results of the regression analysis can be viewed, or other patients' results can be selected.
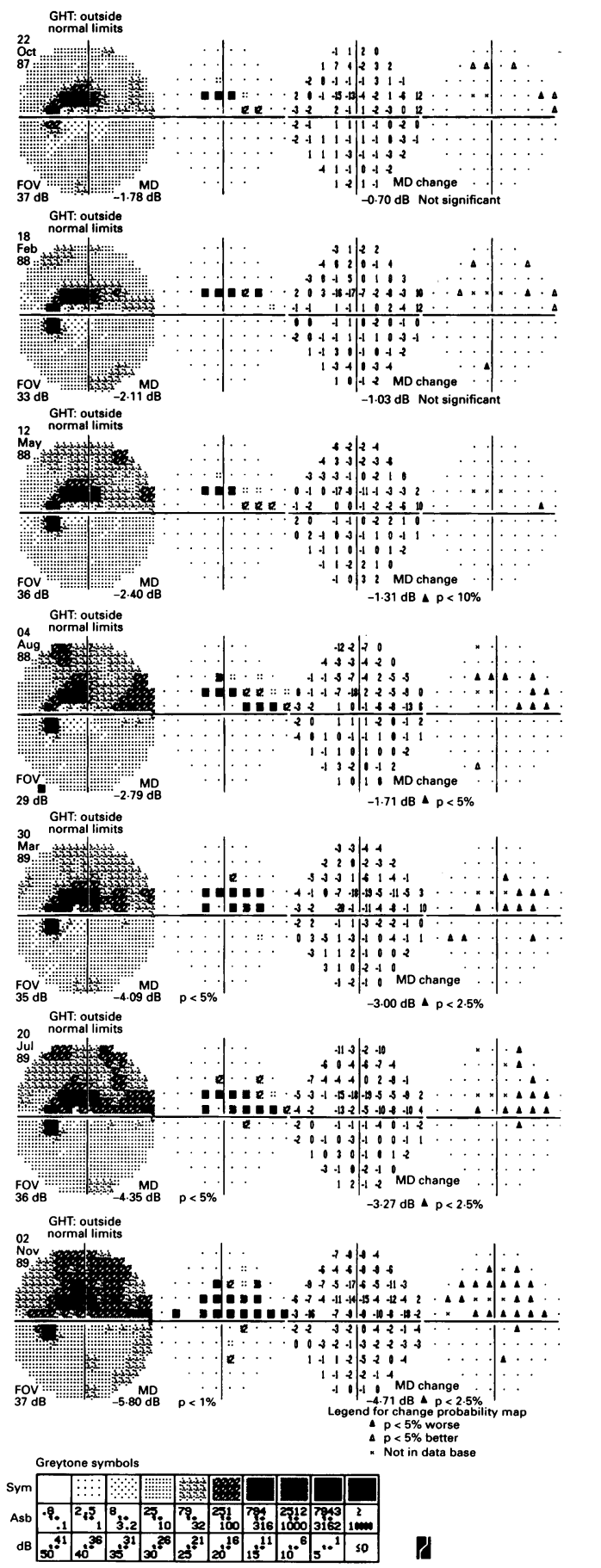

and visual fields are flagged which have different names (to allow checks of misspellings or changes of names), hospital identification numbers, dates of birth, and other inconsistencies. The program allows these common errors in data input to be easily rectified.

\section{PROGRESSOR STATISTICAL ANALYSIS}

Analysis of the entire sequence of visual fields can be made either for an individual patient or automatically for an entire patient group. Automatic analysis is useful for a large database since the calculations may take some time and automatic processing can proceed without supervision to generate the completed analysis for later review.

If patients have undergone testing with different stimulus sizes, then the program will automatically analyse each stimulus size sequence separately. These programs have been designed primarily for use with the HFA $30-2,24-2,30-1$, and 24-1 program visual fields. When visual field sequences include both 30-2 and 24-2 results, these are automatically combined to make use of all the available data in the analysis.

The program will access the database, display all the unique patient names, recall all the patient visual fields, and display them as shown in Figure 1A. The type of display or analysis can be altered and the following refers to the default settings. The patient name is printed on the top left and the dates of testing are printed in a column on the top right. These are printed as visual field number, date, eye $(\mathrm{R} / \mathrm{L})$, foveal threshold, and time in months from the first visual field (for the hard copy plotter outputs only). The $30-2$ visual field locations are maintained in the display and the foveal thresholds and global indices are shown in the top right of the image represented by bars where the length of the bar is proportional to a reference value minus the measured threshold value. A very short bar is nearly normal while a bar of increasing length (going downwards) represents greater sensitivity loss at that visual field location. A calibration marker for $30 \mathrm{~dB}$ is shown on the lower left of the image. At each visual field location the series of bars represents the measured values at the different test dates going from left to right. The separation between bars can be selected for equal spacing or as proportional to time. The scale for time is at the lower left of the figure. In this example the measurements were made at approximately 3 month intervals and the display selected uses equal (unitary) spacing between the bars. However, the statistical analysis uses the actual time scale. It can be seen that thresholds are nearly normal throughout the inferior field while in the 


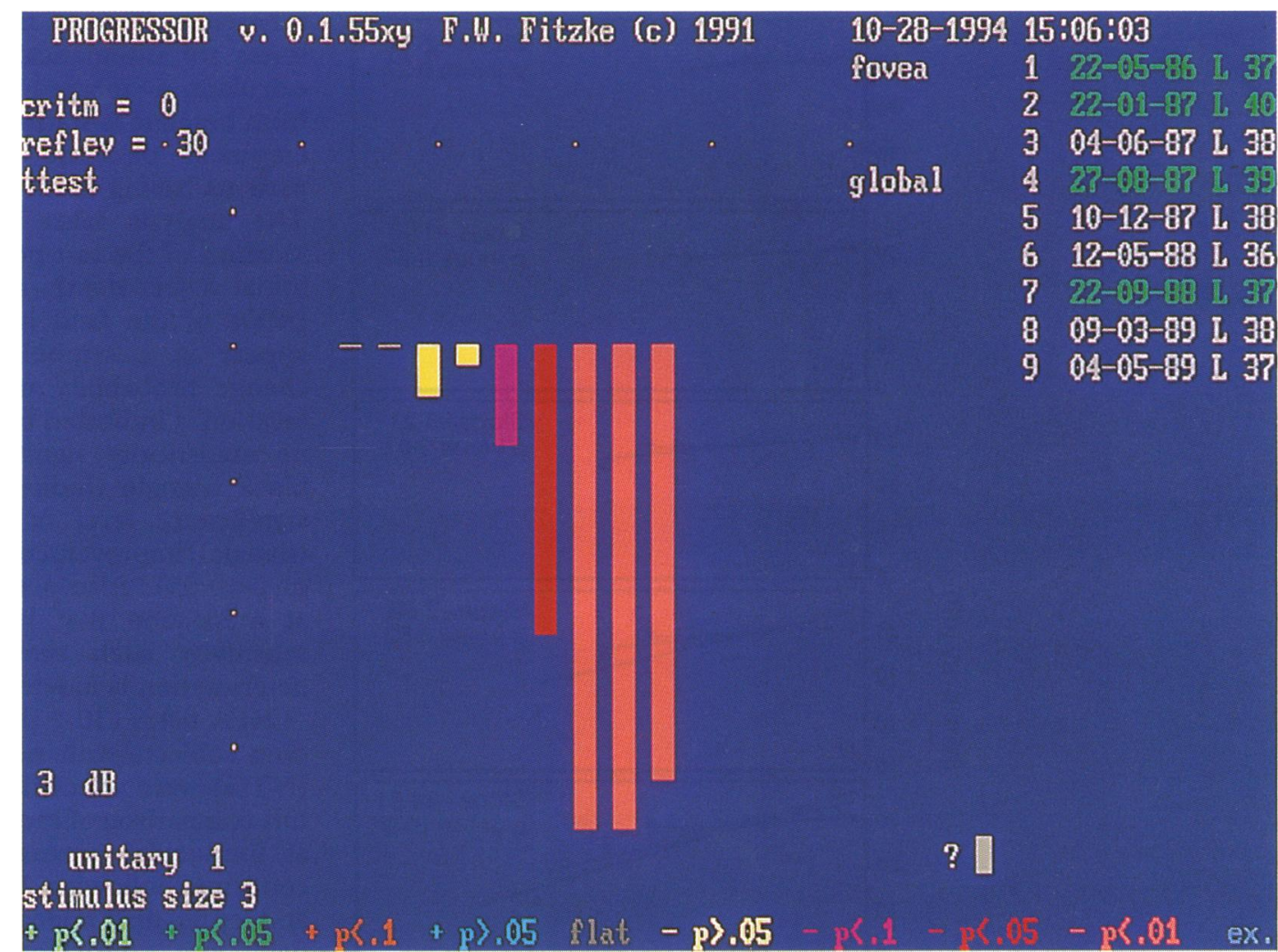

Figure 2 An enlarged version of the results from PROGRESSOR regression analysis at one location (stimulus location 3,-3) from a patient with open angle glaucoma (shown in figure 4). From left to right each bar represents the sensitivity of the location at each of nine sequential fields.

superior field in an area extending from the blind spot to the nasal field there is evidence of visual field loss.

The program applies linear regression analysis to each of the individual field locations to determine whether there is statistically significant progression. (At visual field locations which have repeated measurements the mean of these is used.) Beginning with the third field and sequentially adding subsequent fields linear regression provides values for the slope, or rate of change ( $\mathrm{dB} / \mathrm{years}$ ), by least squares fit. The statistical significance of the critical value of the slope (the magnitude divided by the standard error of the fit) is also generated. Colour coding is now introduced to indicate visually the results of the statistical significance of the fitted slopes. If the slope is less than the criterion value ('flat', default value used $=0 \mathrm{~dB}$ loss per year) then the bar is coloured grey indicating no change. If the slope is negative (indicating loss of retinal sensitivity) with an associated $p$ value of less than 0.01 then the bar is coloured bright red. This indicates highly significant progression. Other significant negative slopes $(p<0.05$ and $p<0.10)$ are highlighted. Negative slopes at locations where the longitudinal sensitivity fluctuation is such that statistical significance is not attained are coloured yellow. Positive slopes (demonstrating improvement, possibly as a result of learning or other factors) are also represented by different colours. A bar coloured cyan indicates a non-significant improvement ( $p>0.05)$ while green indicates significant increases in sensitivity $(p<0.05)$. A bright blue bar indicates a value excluded from the analysis because the value for that location and that visual field was found to lie outside the $2 \mathrm{SD}$ value expected for the entire sequence. This colour coding is summarised on the bottom line of the figure. This analysis is continued and repeated for all test locations for all examination dates and the final graphical display summarises the results.

Figure 2 illustrates the process by showing a magnified version of the analysis at one field location. Figure 3 shows the same data as a sequential plot of the sensitivity $(\mathrm{dB})$ against time of follow up. The first linear regression is initialised after three fields (Figure 3A) giving a negative slope that is, however, not significant $(p=0 \cdot 22)$. Note that the corresponding third bar in the PROGRESSOR output (Figure 2) is therefore coloured yellow. By the fifth field (Figure 3C) the negative slope has increased in magnitude and become statistically significant $(p<0 \cdot 10)$. The rate of loss continues to accelerate consistently and by the final fields in this series the gradient of the negative slope is highly significant $(p<0.01)$ indicated by bright red bars in the PROGRESSOR output.

A difference in variability is reflected in the linear regression analysis where significant progression can be seen even though the losses can be quite small. This is an important component of this analysis because in this way noisy values at locations of high variability do not give rise to significant slopes unless the rate of loss is substantial. Steady, reliable loss, even at a low rate, is immediately evident.

Results from the PROGRESSOR statistical analysis can be further summarised with, for example, histograms to compare different 


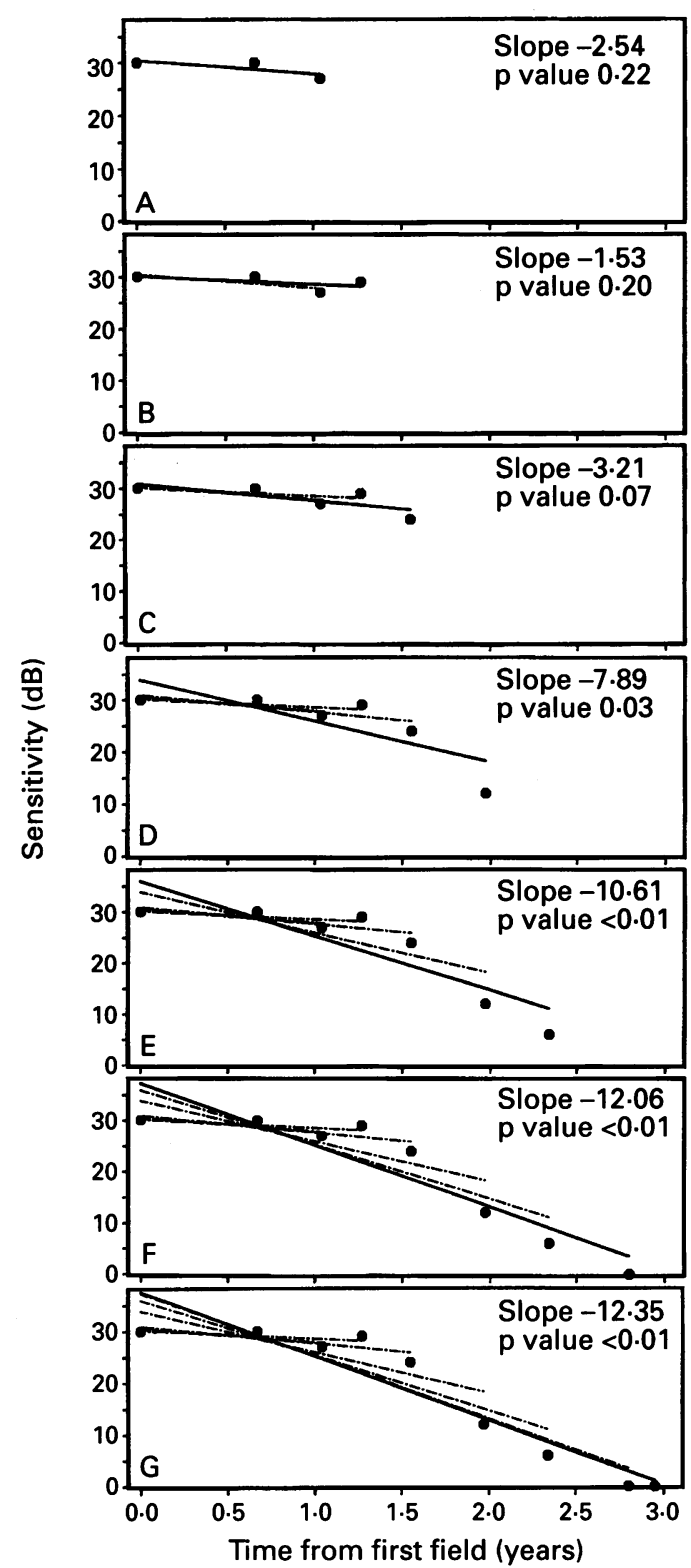

Figure 3 Same data from the example location described in Figure 2 represented in this case as a plot of sensitivity (dB) against time of follow up (years). Each plotting symbol represents the sensitivity value at each field examination. Each solid line represents the updated regression line as each point is added to the series. The first regression analysis is initialised after three fields $(A)$. Note that the magnitude of the gradient of this negative slope increases in time. Each dotted line represents a previous regression line. Statistical significance of the critical value of the slope is also shown.

patients or patient groups. The reference slope, non-linear regression, automatic exclusion of extreme thresholds, spacing bars for different intervals are all options within the analysis. Also, results from the PROGRESSOR analysis can be updated and exported to other programs currently under development that allow further analysis. These include powerful image processing functions ${ }^{26-28}$ that can evaluate spatial characteristics of the field data and also improve the signal to noise ratio of the analysis.

\section{MEASUREMENT OF AGREEMENT BETWEEN} PROGRESSOR AND STATPAC-2 ANALYSIS The STATPAC-2 program $^{7}$ for the HFA includes a glaucoma change probability analysis, in which a follow up field is compared with a baseline. Two of the first three fields in a series are automatically selected and used to calculate a merged baseline. The change from baseline is statistically compared with an empirical database of visual fields from patients having stable glaucomatous field loss. The analysis takes into consideration the location of the test point within the field, the initial defect depth, and the mean deviation (MD) of the field as a whole. The results appear in a symbol form on a glaucoma change probability map (Figure 1B). Each location is indicated by a small dot (indicating no statistically significant change), a solid black triangle (indicating deterioration to a significance level of $\mathrm{p}<0.05$ ), or an open triangle (improvement to a significance level of $p<0.05)$. Also a small cross may appear at a location that had an initial threshold sensitivity such that significance level of deterioration is indeterminate.

HFA fields (30-2 program) of 10 eyes from nine subjects with normal tension glaucoma (NTG) were selected as an initial sample for this comparison of methods. NTG was defined as intraocular pressure (IOP) $<21 \mathrm{~mm} \mathrm{Hg}$ confirmed following 24 hours of IOP phasing, and optic disc appearance and initial visual field loss consistent with a diagnosis of glaucoma. None of the patients underwent any medical or surgical treatment during the study period. The eyes were selected in a nonrandom fashion from a database of 220 NTG patients followed at the glaucoma unit. Each eye had 12 visual fields with similar follow up periods (mean 4.2 (SD 0.5) years) and similar intervals between each field (mean interval $0 \cdot 4$ (SD $0 \cdot 1$ ) years). Eyes were selected that appeared to be progressing on inspection of their STATPAC overview printout. As a global summary the first field for each selected eye had a mean Humphrey mean deviation (MD) of $-6.7 \mathrm{~dB}$ (range -4.4 to $-11.7 \mathrm{~dB}$ ) and mean corrected pattern standard deviation (CPSD) of $7 \cdot 1 \mathrm{~dB}$ (range 3.0 to $13.3 \mathrm{~dB}$ ). STATPAC provides linear regression of each subject's MD against time of follow up. In all selected eyes the rate of $M D$ deterioration was significant at least to the $\mathrm{p}<0.05$ level when compared with Humphrey's normal database.

Each field series was processed by STATPAC2 glaucoma change probability analysis. The printout for each eye was examined and symbols at each location from the final (12th) field were coded and entered manually into the computer for analysis. The peripheral locations of the 30-2 grid (points at 27 degrees eccentricity) and the two locations above and below the blind spot were excluded because of the high threshold variability at these sites. The locations from the STATPAC- 2 printout at the final (12th) field were categorised as progressing or stable with the latter category including locations with higher sensitivity than baseline (open triangles). The progressing criterion for the pointwise linear regression analysis (PROGRESSOR) was defined as a negative slope or rate of loss faster than $1 \mathrm{~dB} /$ year (2 dB/year for outer locations beyond 15 degrees eccentricity) accompanied by a slope 


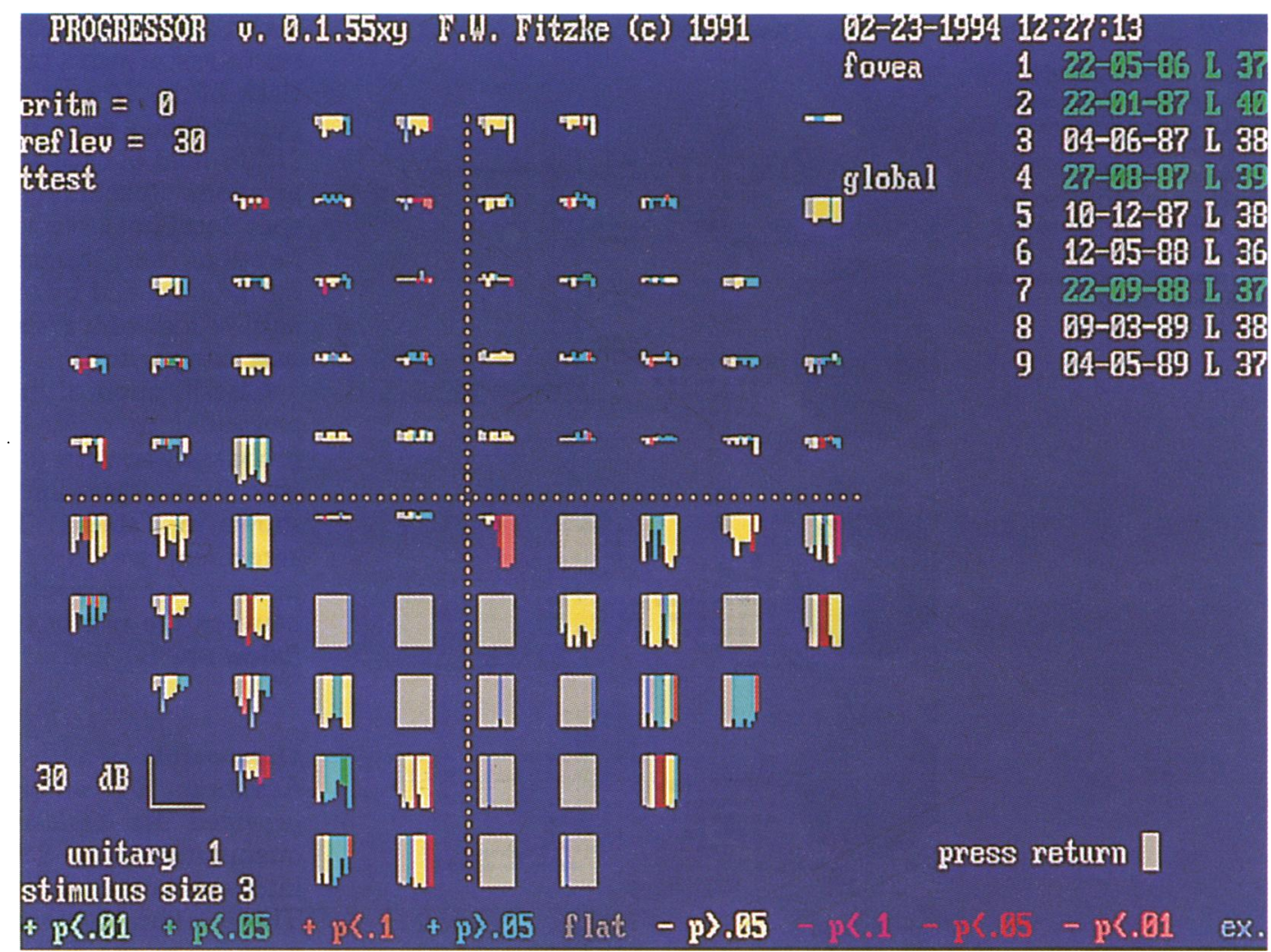

Fig $4 A$

significant at the $\mathrm{p}<0.10$ level at the final (12th) field. This criterion for change had previously been determined by assessing the sensitivity and specificity of the pointwise linear regression technique to predict locations later identified by STATPAC- 2 as unequivocally deteriorated. ${ }^{29}$ Any locations that did not fulfil this progressing criterion were categorised stable by PROGRESSOR. Hence, all locations from the final (12th) field of each series were tabulated in categories of progressing or stable for both the STATPAC-2 and PROGRESSOR analysis. Level of agreement between the two methods was evaluated using a kappa coefficient which gives an indication of agreement beyond chance. ${ }^{30}$

\section{Results}

EXAMPLES OF PROGRESSOR ANALYSIS

The results of the new pointwise linear regression analysis (PROGRESSOR) are illustrated using two example fields. Figure 1A depicts the analysis of a left eye from patient with NTG. In the inferior field which initially had normal thresholds there is no change and after nearly 36 months (nine fields) thresholds remain normal. At the blind spot the expected threshold elevation is generally present. Extending from the blind spot to the region of the nasal step a dense scotoma can be seen. In this region at three locations threshold elevations of about $10 \mathrm{~dB}$ were initially measured which, within a few years, became dense scotomas with elevations of more than $30 \mathrm{~dB}$. These all showed significant losses at $\mathrm{p}<0.05$ before they became absolute scotomas. Interestingly, there are numerous areas extending around this arcuate region which began with normal threshold values but which have gone on to show progression at $\mathrm{p}<0.05$ and $p<0.01$. The three locations which extend along the arcuate region from the scotomas began with normal threshold values but after about 2 years began to show evidence of progression (yellow) which became highly significant (red) and was consistently maintained at about $10 \mathrm{~dB}$ for several fields. Elsewhere throughout the superior field there is further evidence of significant progression (red). For comparison, Figure 1B shows results of the same sequential fields from the same eye as analysed by Humphrey STATPAC2 glaucoma change analysis. This analysis also shows for this example an initial superior arcuate field defect which demonstrates progressive deepening and enlargement with time.

Figure 4B shows output from Humphrey STATPAC-2 analysis of a sequence of nine fields (approximately 36 months' follow up) from a left eye of a subject with open angle glaucoma. The results indicate that the early fields show an initial inferior hemifield defect which appears stable in depth and extent over time. The consistent presence of a black (filled) triangular symbol close to fixation in the inferior hemifield (at stimulus coordinates $3,-3)$ in the last four fields within the sequence does indicate, however, localised progression at this site. For comparison, results of pointwise PROGRESSOR regression analysis of the same fields are shown in Figure 4A. This display also clearly shows the initial absolute field defect involving most of the locations in the inferior hemifield indicated by unchanging grey bars of maximum length. 
Fig $4 B$

Figure 4 Analysis of a series of visual field results from a patient with open angle glaucoma. (A)

Shows the results as displayed by PROGRESSOR. For comparison (B) shows the same data as described by output from STATPAC-2 glaucoma change probability analysis.
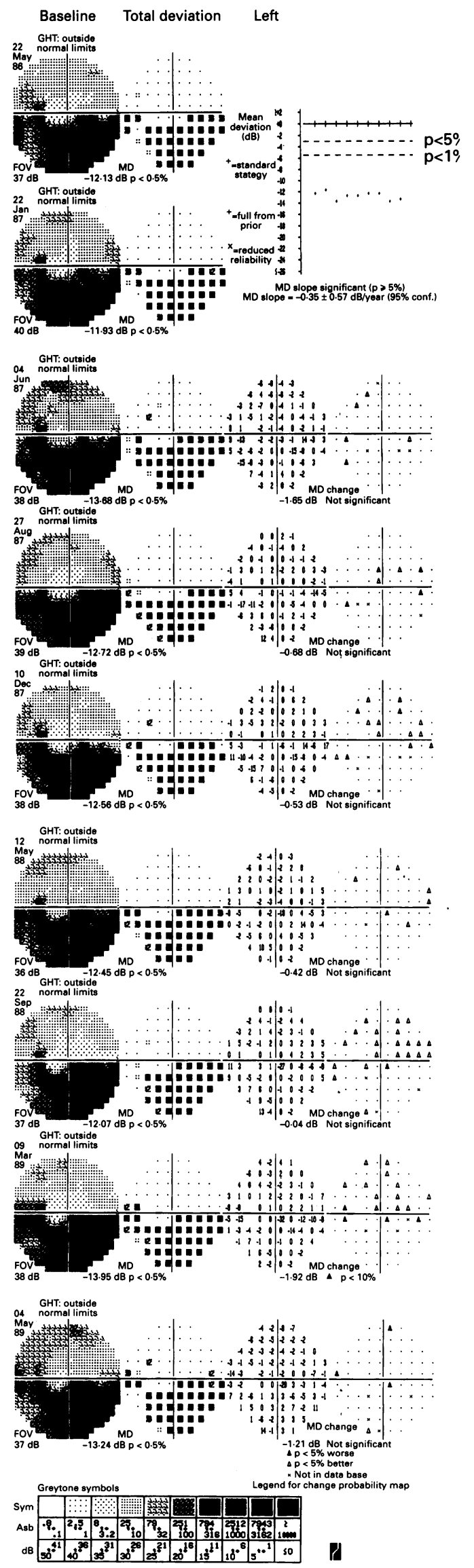

The colour coding does also highlight the location close to fixation in the inferior hemifield which demonstrates marked, highly significant $(p<0.01$, bright red) deterioration in sensitivity.
MEASUREMENT OF AGREEMENT BETWEEN

PROGRESSOR AND STATPAC-2 ANALYSIS

Each of the 10 eyes from the sample of NTG patients had a series of 12 fields. From the final (12th) field of each series a total of 540 retinal locations (excluding peripheral and blind spot locations) were analysed. A further 49 locations were excluded because they could not be classified by STATPAC-2 (indeterminate level of loss with respect to baseline and database comparison).

Classification of the remaining locations as progressing or stable by the glaucoma change probability analysis (STATPAC-2) and the pointwise linear regression analysis (PROGRESSOR) is shown in Table 1. The kappa coefficient of agreement was 0.62 (SE 0.04 ). This value indicates a reasonably good level of agreement between the two techniques for this length of follow up. ${ }^{31}$

\section{Discussion}

Advances in automated perimetry have provided the clinician with vast amounts of quantitative visual field data from which clear interpretation and diagnosis must be formed. This is a formidable task which is further compounded by the difficulty in detecting whether differences between serial visual fields are real or merely fluctuation as a result of patient response variability or other factors.

Summary measures or global estimates of sensitivity change are insensitive in detecting subtle and localised glaucomatous field loss against the background of the noisy perimetric process. Evaluation of sensitivity at individual locations to identify change more precisely has therefore been adopted by recent methods of analysis such as STATPAC- 2 glaucoma change probability analysis for Humphrey data. ${ }^{7}$ It generates probability estimates of individual locations changing in sensitivity when compared with baseline and normal reference data and has been shown to demonstrate differences between static and deteriorating field locations. ${ }^{32}$ Another study indicated good agreement between this technique and clinical impressions of visual field change. ${ }^{33}$

Results from an initial sample of series of glaucomatous fields indicate reasonably good agreement between the STATPAC- 2 technique and the new pointwise linear regression method to separate progressing from stable locations. The differences in classification between the methods can be explained by various factors. For example, the STATPAC-2 procedure

Table 1 Number of locations from final (12th) field of all series found progressing or stable by glaucoma change probability analysis (STATPAC-2) and pointwise linear regression technique (PROGRESSOR). Excludes 49 locations classified as indeterminate by STATPAC-2 analysis

\begin{tabular}{c|c|c|c|c|}
\hline \multicolumn{5}{c}{ STATPAC-2 criteria } \\
\cline { 2 - 5 } & & Progressing & Stable & Totals \\
\cline { 2 - 5 } $\begin{array}{c}\text { PROGRESSOR } \\
\text { criteria }\end{array}$ & Progressing & 106 & 41 & 147 \\
\cline { 2 - 5 } & Stable & 39 & 305 & 344 \\
\cline { 2 - 5 } & Totals & 145 & 346 & \\
\hline
\end{tabular}


represents an event type analysis which may be particularly sensitive to sudden, marked loss in sensitivity relative to baseline. Conversely, the regression technique is a trend type analysis which should detect more subtle gradually developing loss. The two methods are perhaps correctly identifying different progressing locations in the same field which show a decay pattern to which the respective analysis method is more sensitive. Also the STATPAC- 2 analysis requires different levels of deterioration for different locations within the field. For example, a greater level of loss is required to give a statistically significant change in areas of higher threshold variability. The pointwise linear regression technique is not weighted in this fashion but rather identifies significant values of field loss in relation to long term fluctuation. Additionally, this pointwise linear regression technique of determining the rate of loss at individual locations against time of follow up uses all the data from a complete series with less dependence on baseline and normal reference data. In fact, extracting and quantifying this pointwise rate of field loss alone may provide clinically useful information. ${ }^{34}$

It should be noted that the sensitivity values of field locations are, however, not independent in time or space. Furthermore, using multiple linear regression analyses at each and every field location inflates the estimation error used for determining the significance of a slope and subsequently affects the interpretation of the associated $p$ value. These are violations of the statistical assumptions that underpin this type of pointwise linear regression analysis. However, the $p$ values are simply used as arbitrary cut off values to decide if the rate of sensitivity loss at an individual location is significant. These cut off values can be altered and set within the analysis software to accommodate clinically based decision criteria. Different values may, for example, be used in different areas of the field because variability of sensitivity values is partly a function of location and eccentricity. Indeed, by adjusting these arbitrary cut off values we can maximise the sensitivity and specificity of the technique to detect future field progression. ${ }^{29}$

The PROGRESSOR software employs a method of pointwise linear regression and provides a graphical display of results using colour coding to clearly indicate significant field progression. This may prove easier to interpret than inspection of long 'print outs' of field series. Furthermore, this method allows the clinician to examine the spatial configuration of field loss and damage. Such analysis of spatial patterns of loss is intrinsic to the detection of true glaucomatous progression. The sensitivity values of neighbouring field locations have been shown to be spatially dependent ${ }^{35}$ and the sensitivity of detecting glaucomatous loss has been improved by incorporating spatial information into certain forms of visual field analysis. ${ }^{36}$ Some, newer methods of analysis are wholly dependent on spatial properties of the visual field. ${ }^{37}$

In addition to the novel spatial presentation of field loss central to the PROGRESSOR statistical analysis, the software has been developed to allow exportation of data to other programs that integrate spatial factors into the process of detecting field progression. These methods take advantage of the recognised spatial correlation of the data and, by utilising image processing techniques, effectively characterise spatial properties of field data. ${ }^{26}$ They have also been shown to quantify and reduce the variability inherent in these data thus potentially improving the signal to noise ratio of visual field analysis. ${ }^{27} 28$ Moreover, these image processing techniques have been shown to improve the pointwise linear regression algorithm for predicting and detecting glaucomatous field loss. ${ }^{38}$

In summary, the PROGRESSOR software package implements a new method of analysis, based on pointwise linear regression, that transforms the field data into a colour coded visual form which combines spatial relations with temporal change. The agreement between the pointwise linear regression method implemented by PROGRESSOR and the glaucoma change probability analysis from STATPAC- 2 to separate progressing from stable retinal locations appears to be good using series of fields from a sample of normal tension glaucoma patients. The PROGRESSOR analysis, using one colour coded figure, may prove easier to interpret than the long print out of small triangular symbols provided by STATPAC-2. We are currently investigating this potential advantage by evaluating clinicians' interpretation and agreement using PROGRESSOR analysis. Further developments to add to the suite of programs already provided are under way. These allow spatial relations to be characterised in a quantitative way and are used to reduce noise and improve the detection of true change. The software has already been extensively used and tested on hospital databases consisting of many thousands of patient visual fields. By allowing visual field results to be presented in a form which is visually and intuitively easy to interpret the methods presented here may provide an efficient device for detecting true progression in glaucomatous field loss.

Supported in part by grants from the National Retinitis Pigmentosa Foundation, the Medical Research Council, the Wellcome Trust, and the International Glaucoma Association.

A I McNaught was supported by the Friends of Moorfields A I McNaught was supported by the Friends of Moorfields
and D P Crabb is supported by the Royal National Institute for and D P C

the Blind. the PROGRESSOR figures. The Institute of Ophthalmology retains the intellectual property rights to the PROGRESSOR software described in this paper.

1 Zulauf M, Caprioli J. What constitutes progression of glaucomatous visual field defects? Surv Ophthalmol 1992; glaucomato $130-46$.

2 Hoskins HD. Does computerised perimetry offer practical advances in choice of therapy in the glaucoma patient? Eye 1992; 6: 43-6.

3 Mikelberg F. Do computerised visual fields and automated optic disc analysis assist in the choice of therapy in glaucoma? Eye 1992; 6: 47-9.

4 Hitchings RA. Psychophysical testing in glaucoma. $\mathrm{Br} \mathfrak{f}$ Ophthalmol 1993; 77: 471-2.

5 Johnson CA. Modern developments in clinical perimetry. Curr Opin Ophthalmol 1993; 4: 7-13.

6 Fitzke FW, McNaught AI. The diagnosis of visual field progression in glaucoma. Curr Opin Ophthalmol 1994; 5: $110-5$.

7 Heijl A, Lindgren G, Lindgren A, Olsson J, Asman P, Myers S, et al. Extended empirical statistical package for evaluation of single and multiple fields in glaucoma: STATPAC-2. In: Mills RP, Heijl A, eds. Perimetry updace 
1990/91. Amsterdam: Kugler and Ghedini, 1991: 303-15.

8 Bebie H, Fankhauser F. Delta manual. Zurich: Schlieren Interzaeg AG, 1982

9 Holmin C, Krakau C. Regression analysis of the central field in chronic glaucoma cases. Arch Ophthalmol 1982; 60: 267-74.

10 Wu D, Schwartz B, Nagin P. Trend analyses of automated visual fields. Doc Ophthalmol Proc Ser 1987; 49: 175-89.

11 O'Brien C, Schwartz B, Takamoto T, Wu D. Intraocular pressure and the rate of visual field loss in chronic openangle glaucoma. Am f Ophthalmol 1991; 111: 491-500.

12 Crick R, Newson R, Shipley M, Blackmore H, Bulpitt C. The progress of the visual field in chronic simple glaucoma and ocular hypertension treated topically with pilocarpine or with timolol. Eye 1990; 4: 563-71.

13 Wegner A, Ugi I, Hofman A. A long-term visual field evaluation of glaucoma patients treated topically with timolol or carteolol. In: Mills RP, ed Perimetry update 1992/93. Amsterdam: Kugler and Ghedini, 1993: 143-5.

14 Weber J, Koll W, Krieglstein G. Intraocular pressure and visual field decay in chronic glaucoma. German $f$ Ophthalmol 1993; 2: 165-9.

15 Vogel R, Crick R, Mills K, Reynolds P, Sass W, Clineschmidt C, et al. Effect of timolol versus pilocarpine on visual field progression in patients with primary openangle glaucoma. Ophthalmology 1992; 99: 1505-11.

16 Mikelberg F, Drance S. The mode of progression of visual field defects in glaucoma. Am $\mathcal{f}$ Ophthalmol 1984; 98: 443-5.

17 Hoskins HD, Jensvold N, Zaretsky M, Hetherington J. Rate of progression of discrete areas of the visual field. In: Heijl A, ed. Perimetry update 1988/89. Amsterdam: Kugler and A, edini, 1989: 173-6.

18 O'Brien C, Schwartz B. The visual field in chronic open angle glaucoma: the rate of change in different regions of the field. Eye 1990; 4: 557-62.

19 Wild JM, Hussey MK, Flanagan JG, Trope GE. Pointwise topographical and longitudinal modeling of the visual field in glaucoma. Invest Ophthalmol Vis Sci 1993; 34: 1907-16.

20 Wild JM, Hussey MK, Flanagan JG, Trope GE. Forecasting progression of glaucomatous visual field loss. In: Mills RP, ed. Perimetry update 1992/93. Amsterdam: Kugler and Ghedini, 1993: 89-102.

21 Noureddin B, Poinoosawmy D, Fitzke FW, Hitchings RA. Regression analysis of visual field progression in low tension glaucoma. Br f Ophthalmol 1991; 75: 493-5.

22 Poinoosawmy D, Wu J, Fitzke FW, Hitchings RA. Discrimination between progression and non-progression visual field loss in low tension glaucoma using MDT. In Mills RP, ed. Perimetry update 1992/93. Amsterdam: Kugler and Ghedini, 1993: 109-14.

23 O'Brien C, Schwartz B. Point by point linear regression analysis of automated visual fields in primary open-angle glaucoma. In: Mills RP, ed. Perimetry update 1992/93. Amsterdam: Kugler and Ghedini, 1993: 149-52.

24 Wild JM, Hutchings N, Hussey MK, Flanagan JG, Trope GE. Univariate linear regression of pointwise visual field sensitivity against time of follow-up. ARVO abstracts. Invest Ophthalmol Vis Sci 1994; 35 (suppl): 4306.

25 McNaught AI, Crabb DP, Fitzke FW, Hitchings RA. Modelling sensitivity loss in low tension glaucoma. ARVO abstracts. Invest Ophthalmol Vis Sci 1994; 35 (suppl): 4295. 26 Crabb DP, Fitzke FW, Edgar DF, McNaught AI. Improving the analysis of glaucomatous visual field data with image processing techniques. ARVO abstracts. Invest with image processing techniques. ARVO a

27 Fitzke FW, Crabb DP, McNaught AI, Edgar DF, Hitchings $\mathrm{RA}$. Image processing of visual computerised visual field data. Br f Ophthalmol 1995; 79: 207-12.

28 Crabb DP, Fitzke FW, Edgar DF, McNaught AI, Wynn HP. New approach to estimating the variability in visual field data using an image processing technique. $\mathrm{Br} \mathcal{F}$ Ophthalmol 1995; 79: 213-7.

29 McNaught AI, Crabb DP, Fitzke FW, Hitchings RA. Early diagnosis of glaucomatous progression: comparison of pointwise regression analysis and current methods. pointwise regression analysis and current methods. meeting, Rejykavik, Iceland. July, 1993.

30 Fleiss JL. Statistical methods for rates and proportions. New York: John Wiley, 1981: 212-25.

31 Landis JR, Koch GG. The measurement of observer agreement for categorical data. Biometrics 1977; 33: 159-74.

32 Morgan RK, Feuer WJ, Anderson DR. Statpac2 glaucoma change probability. Arch Ophthalmol 1991; 109: 1690-2.

33 Tuulonen A, Airaksinen PJ. Statpac2 compared to clinical evaluation of visual fields. In: Mills RP, Heijl A, eds. Perimetry update 1990/91. Amsterdam: Kugler and Perimetry update 19901

34 McNaught AI, Crabb DP, Fitzke FW, Hitchings RA. Modelling series of visual fields to detect progression in normal tension glaucoma. Graefes Arch Clin Exp Ophthalmol 1995 (in press).

35 Lachenmayr B, Kiermeir U, Kojetinsky S. Neighbouring points of a normal visual field are not statistically independent. ARVO abstracts. Invest Ophthalmol Vis Sci 1994; 35 (suppl): 313

36 Asman P, Heijl A, Olsson J, Rootzen H. Spatial analyses of the glaucomatous visual field: a comparison with traditional field indices. Arch Ophthalmol 1992; 70: 679-86.

37 Spenceley SE, Henson DB, Bull DR. Visual field analysis using artificial neural networks. Ophthalmol Physiol Opt 1994; 14: 239-45.

38 Crabb DP, McNaught AI, Fitzke FW, Hitchings RA. Spatially enhanced modelling of sensitivity decay in low tension glaucoma. In: Mills RP, ed Perimetry update 1994/95. Amsterdam: Kugler and Ghedini, 1995: 73-81. 